\title{
Molecular lodine - An Efficient Oxidative Reagent for Aromatization of Trifluoromethyl Substituted Chromenones
}

\author{
Jussara Navarini (PG), Helio G. Bonacorso* (PQ), Carson W. Wiethan (PG), Rosália \\ Andrighetto (PG), Marcos A. P. Martins (PQ), Nilo Zanatta
}

\author{
Núcleo de Química de Heterociclos (NUQUIMHE), Departamento de Química, Universidade Federal de \\ Santa Maria, 97105-900, Santa Maria, RS. *E-mail heliogb@base.ufsm.br
}

Keywords: Molecular lodine, Chromenones, Oxidative Aromatization

\section{INTRODUCTION}

Functionalized chromenes and benzopyranes are important compounds which, due to their biological activity, find wide application in medicinal chemistry. They display not only spasmolytic, diuretic, clotting, antiviral, anti-tumoral and anti-anaphylactic activity, but can also be used as pigments, photo-active materials and biodegradable agrochemicals. ${ }^{1,2}$

The use of molecular iodine as an oxidant to promote aromatization of cyclohexanone derivatives was first reported in 1980 by Tamura and Yoshimoto ${ }^{3}$. In recent years, molecular iodine has received considerable attention as an inexpensive, non-toxic, readily available oxidant to promote aromatization of cyclohexanone derivatives and their heterocyclic analogues. ${ }^{4,5,6}$ In this context, herein we describe the synthesis of 5-alkoxy-3,4-dihydro-2Hchromenes, using as starting material the trifluoromethylated chromenones recently reported by our research group. ${ }^{7}$

\section{RESULTS AND DISCUSSION}

The reactions so far investigated can be seen in Scheme 1. Initially, the reactions of compounds 1 and $\mathrm{MeOH} / \mathrm{I}_{2}$ under reflux were carried out for 16-24 hours, which led to formation of compounds 2 in 65-89\% yields. Subsequently, aromatization reactions of the chromenones were performed using different alcohols (ethanol, $n$ propanol and benzilic alcohol), where $\mathbf{A r}=\mathrm{Ph}$ and $\mathbf{R}=\mathrm{Me}$ (Scheme1). Only reactions using ethanol and $n$-propanol led to derivatives 2; for the other alcohols the starting material was recovered.

The chromenes 2 were purified by column chromatography using hexane/ethyl acetate (4:1) as eluent and characterized by NMR ${ }^{1} \mathrm{H}$, and ${ }^{13} \mathrm{C}$ and GC/MS spectrometry.

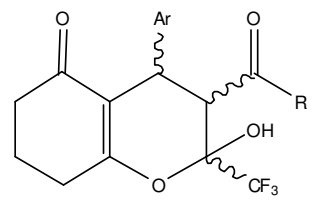

1

i: $\mathrm{I}_{2}$ (2 equiv.), $\mathrm{R}^{1} \mathrm{OH}, 70^{\circ} \mathrm{C}, 16-24 \mathrm{~h}$

$\mathrm{Ar}: \mathrm{Ph}, 4-\mathrm{NO}_{2} \mathrm{Ph}, 4-\mathrm{MeOPh}$

$\mathrm{R}: \mathrm{Me}, \mathrm{Ph}, 2$-Furyl

$\mathrm{R}^{1}: \mathrm{CH}_{3}, \mathrm{C}_{2} \mathrm{H}_{5}, n-\mathrm{C}_{3} \mathrm{H}_{7}$

Scheme 1: Synthesis of 5-alkoxy-3,4-dihydro- $2 \mathrm{H}$ chromenes (2)

\section{CONCLUSION}

The methodology described, $\mathrm{I}_{2} / \mathrm{R}^{1} \mathrm{OH}$, was efficient and versatile for obtaining of 5-alkoxy-3,4-dihydro$2 \mathrm{H}$-chromenes 2, which contain a benzo[b]pyran, which contain an alkoxy substituent at C-5 derived from the employed alcohol.

\section{ACKNOWLEDGEMENTS}

\section{CNPq-CAPES-FATEC}

\section{REFERENCES}

(a) Foye, W. O. Prinicipi di Chemico Farmaceutica; Piccin: Padova, Italy, 1991; pp. 416; (b) Andreani, L. L.; Lapi, E. Bull. Chim. Farm. 1960, 99, 583; (c) Zhang, Y. L.; Chen, B. Z.; Zheng, K. Q.; Xu, M. L.; Lei, X. H. Yao Xue Xue Bao. 1982, 17, 17; Chem. Abstr. 1982, 96, 135383e; (d) Witte, E. C.; Neubert, P.; Roesch, A. Ger. Offen. DE, 1986; Chem. Abstr. 1986, 104, 224915f; (e) Bonsignore, L.; Loy, G.; Secci, D.; Calignano, A. Eur. J. Med. Chem. 1993, 28, 517.

${ }^{2}$ Zhong, W.; Zhao, Y.; Su, W. Tetrahedron, 2008, 64, 5491

${ }^{3}$ Tamura, Y.; Yoshimoto, Y. Chem. Ind. 1980, 888.

${ }^{4}$ Mphahlele, J. M. Molecules, 2009, 14, 5308.

${ }^{5}$ Banerjee, A. K.; Vera, w.; Mora, H.; Laya, M. S.; Bedoya, L.; Cabrera E. V. J. Sci. Ind. Res. 2006, 65, 299.

${ }^{6}$ Mphahlele, J. M.; Moeka, B. T. Org. Biomol. Chem. 2005, 3, 2469.

Bonacorso, H. G.; Navarini, J.; Wiethan, C. W.; Bortolotto, G. P.

Paim, G.R.; Cavinatto, S.; Martins, M.A.; Zanatta, N.; Caro, M. S.

B. J. Fluorine Chem. 2011, 132, 166. 